\title{
POSITRON FOCUSING IN THE ADVANCED PHOTON SOURCE (APS)

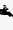 LINEAR ACCELERATOR*
}

\author{
Y. L. Qian and M. White, Argonne National Laboratory, Argonne, IL, 60439 USA
}

\section{Abstract}

Positrons are created by a bremsstrahlung shower process, and are therefore produced with broad divergence and a large energy spread. The $e^{+} / e^{-}$conversion ratio is on the order of $1 / 200$, so the positron focusing system is critical to ensure good positron capture and transport efficiency. The positron focusing system is described, and functions of the different magnetic elements are discussed. Some improvements to the focusing system are suggested, although the linac's design positron intensity of $8 \mathrm{~mA}$ has already been achieved [1].

\section{INTRODUCTION}

Positrons are used in the APS storage ring in order to avoid ion trapping. Electrons are accelerated to $200 \mathrm{MeV}$ in the electron linac, and focused to a 3-mm to 5-mm-diameter spot on a 2-radiation-length-thick tungsten target. The energy spectrum of positrons emerging from the target is shown in Figure 1. The pair-produced positrons (and electrons) are then accelerated to $450 \mathrm{MeV}$ by the remainder of the linac. Positrons are produced with divergent angles, a broad energy spectrum, and with a much larger emittance than that of the initial electron beam; thus the positron linac focusing system is different from the electron linac focusing system. A short pulsed solenoid serves as first part of the focusing system for the low energy positron beam, followed by $6 \mathrm{~m}$ of $\mathrm{DC}$ solenoids. Quadrupole magnets focus the positrons as they gain energy and contain them through the remainder of the linac.

\section{SOLENOID FOCUSING}

A 5-cm-long pulsed solenoidal coil is the first focusing element in the positron linac. The coil has an inner diameter of $25 \mathrm{~mm}$ and operates at its design peak current of $5000 \mathrm{~A}$. It is fabricated from a single piece of water-cooled copper tubing that is wound around the inside and the outside of a cylindrical piece of ceramic. There are a total of 16 turns. The tube rests in grooves machined into the inside and outside of the ceramic. The ceramic support is located between the two coil layers to provide mechanical stability. A prototype made with machinable ceramic was used in the first tests, but the ceramic did not withstand the high radiation fields and was replaced with $99.5 \%$ pure ceramic [2]. The layout of the pulsed coil and target is shown in Figure 2.

The pulsed solenoid coil serves as a quarter-wave transformer (QWT) for the beam's phase space. It rotates the initial transverse phase space from a small radius and a large slope to a phase space with a larger radius and a smaller slope.

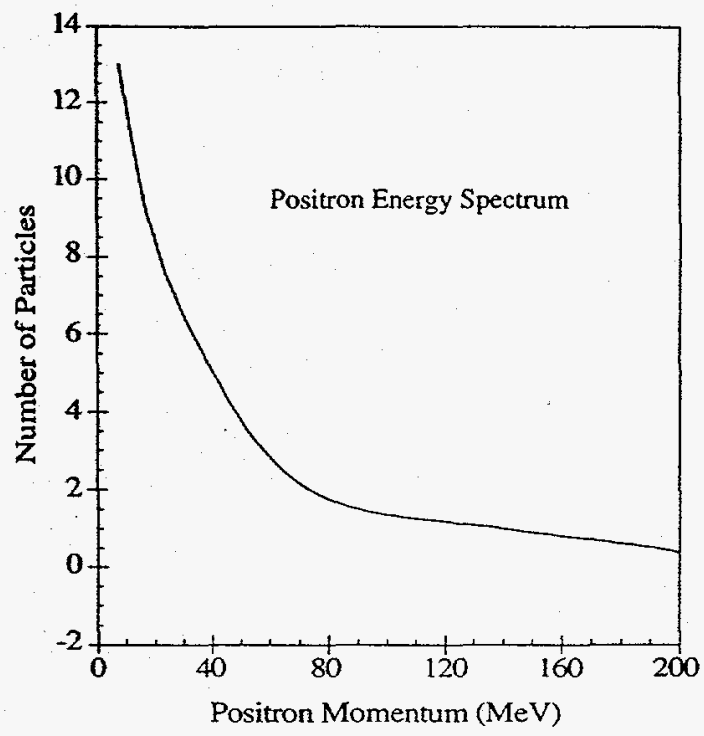

Figure 1: Momentum spectrum of positrons from the target.

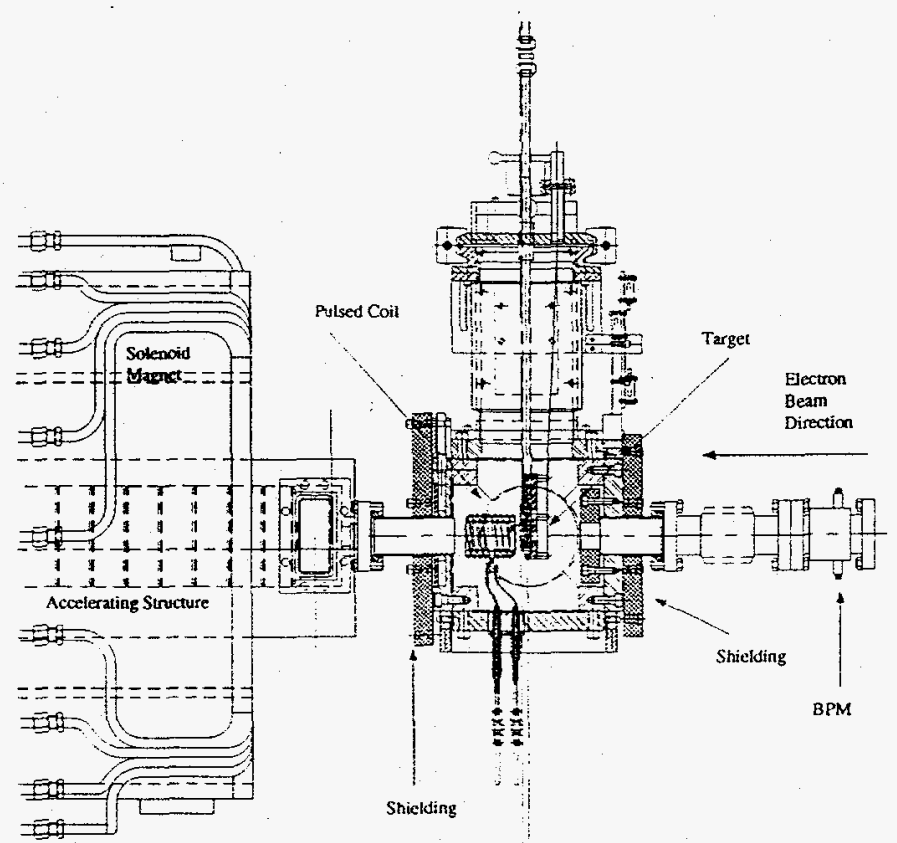

Figure 2: Layout of the pulsed coil and positron target.

The DC solenoidal field is generated by two 2.5 -m-long solenoid magnets with a 0.2 -m-iong solenoid coil between them for matching. The long solenoids have 784 turns in four

\footnotetext{
"Work supported by the U. S. Department of Energy, Office of Basic Sciences under the Contract W-31-109-ENG-38 


\section{DISCLAIMER}

Portions of this document may be illegible in electronic image products. Images are produced from the best available original document. 
layers with integrated steering and correction coils. The maximum axial field of the long solenoids at $1000 \mathrm{~A}$ is $0.36 \mathrm{~T}$.

The acceptance, $A$, is determined by the field in the long solenoids and the aperture of the accelerating structure:

$$
\mathrm{A}=\frac{\pi}{2} e B_{2} r_{2}^{2}
$$

where $e$ is the charge of the particle, $B_{2}$ is the longitudinal DC field in the solenoid, and $r_{2}$ is the radius of the aperture. The minimum radius of the accelerating structures is $9 \mathrm{~mm}$; therefore the acceptance is about $\mathrm{A}=0.3 \pi \mathrm{MeV} / \mathrm{c} \mathrm{cm}$. The maximum transverse momentum, $P_{r}$, that can be captured from the target is determined by

$$
P_{r}=\frac{A}{r_{1}}
$$

where $r_{1}$ is the beam-spot radius on the target. With a 1.5mm-radius beam spot on target, a maximum $P_{r}$ of $2 \mathrm{MeV} / \mathrm{c}$ is accepted into the accelerating structure. Equation (2) shows that a small spot size on the target is an advantage since more particles with higher $P_{r}$ are accepted.

The matching condition for the two solenoids is:

$$
B_{1}=\frac{r_{2}}{r_{1}} B_{2} \text {. }
$$

The optimized length of the QWT pulsed solenoid coil [3] is given by

$$
L=\frac{\pi r_{2}^{2}}{A} P_{z}
$$

$P_{z}$ is $8 \mathrm{MeV}$, so a 5 -cm-long pulsed solenoid coil was used The longitudinal momentum acceptance of the QWT is

$$
\frac{\Delta P}{P} \approx C \frac{B_{2}}{B_{1}}
$$

where $\mathrm{C}$ is a geometric constant on the order of one. Positrons with longitudinal momentum $8 \pm 1.6 \mathrm{MeV} / \mathrm{c}$ are captured and accelerated. Path lengths of the particles in the QWT depend on $P_{r}$. Differences in momenta lead to bunch lengthening that eventually limits the acceptance. Ideally $B_{1}$ is constant and the phase difference between a particle of zero transverse momentum and transverse momentum $P_{r}$ is given by [4]

$$
\Delta \varphi=\frac{360^{\circ}}{\lambda_{r f}} L\left(\sqrt{\left(\frac{P_{r}}{P_{z}}\right)^{2}+1}-1\right) .
$$

In our case $\lambda_{r f}=10.5 \mathrm{~cm}, \mathrm{~L}=5 \mathrm{~cm}, P_{r}=2 \mathrm{MeV} / \mathrm{c}, P_{z}=8$ $\mathrm{MeV} / \mathrm{c}$, and the phase difference is $\Delta \varphi=5.5^{\circ}$.

The emittance at the exit of a long solenoid is reduced, and that is an advantage in beam transport. The accepted beam emittance at the surface of the target is $1.5 * 240 \pi \mathrm{mm} \mathrm{mrad}$. The pulsed solenoid changes the emittance at the entry of the long solenoid region to $9 * 40 \pi \mathrm{mm}$ mrad. Subsequent acceleration increases the beam energy and reduces its emittance. The longitudinal momentum is $\approx 100 \mathrm{MeV} / \mathrm{c}$ after the $7-\mathrm{m}$-long solenoid region, and the emittance is reduced to 9*4 $\pi$ mm mrad.

Solenoids are used to focus the low energy positrons in both transverse planes simultaneously, but they do not really reduce the radial beam size. Perturbations or instabilities can lead to beam losses in a long solenoid region, because the transverse beam size is the same as the accelerating structure aperture. The actual beam size just downstream of the DC solenoids is almost the size of the aperture when observed on a fluorescent screen. The transverse beam size downstream of the target must be decreased as early as possible to reduce beam loss, and that is done by quadrupole magnets. Many other positron sources use quadrupoles from a beam energy of about $50 \mathrm{MeV},[5-7]$ and we feel that a shorter solenoid region could improve the $e^{+}$yield here as well. KEK experienced an increase in their $e^{+} / e^{-}$ratio with a shorter solenoid in their positron linac [7].

Both positrons and electrons are captured and are accelerated in the positron linac, as this linac is not equipped with a chicane that removes the electrons at low energy. Optimizing the positron beam is complicated by the presence of both positive and negative particles. The beam position monitors are not able to distinguish the sign of the charge and cannot be used independently to steer the positrons. However, six fluorescent screens in the positron linac are successfully used in combination with the BPM information.

\section{QUADRUPOLE FOCUSING}

Quadrupoles that focus the beam and reduce its radial size are the next part of the positron focusing system. The radial beam size should be reduced as soon as practical after the beam exits the solenoidal field. The best way to accomplish that would have been with a small-aperture quadrupole doublet in the 0.5-m-long drift space downstream of the solenoids. The $38-\mathrm{mm}$-diameter vacuum pipe in the drift spaces is larger than the accelerating structure aperture, and a doublet in that location would reduce the beam size to the extent that the downstream quadrupoles would not cause further beam loss. Without this doublet, the transverse beam size is defocused in one plane by the quadrupoles and easily becomes larger than the aperture of the accelerating structure.

Twenty-three 152-mm-diameter, 300-mm-long largeaperture quadrupole magnets are installed over the accelerating structures in the positron linac. A single large-aperture quadrupole that is only $150 \mathrm{~mm}$ long is intended to match the solenoidal field to the quadrupole field before the transverse beam size has been reduced much. This focusing/defocusing action probably leads to some beam loss in our case, since the first quadrupole occurs where the beam size is still large. The large quadrupole aperture does offer flexibility in magnet placement and allows us to use most of our drift spaces for diagnostics, such as fluorescent screens, current monitors, and BPMs. The required distance between the quadrupoles is determined in the thin lens approximation by [5] 


$$
L=\frac{\pi r_{2}^{2}}{A} P_{z}
$$

More quadrupoles are required at the low energy end than at high energy, so the quadrupole spacing on the first accelerating structure after the solenoids is short. Largeaperture quadrupoles on the first two accelerating structures after the solenoids still provide a reasonable solution for focusing the beam without the use of short accelerating structures.

The positron linac beam envelope was calculated for the existing focusing system using TRANSPORT [8] and is shown in Figure 3 . The beam loss at the first accelerating structure after the solenoid could be eliminated by adding a doublet of small-aperture quadrupoles in the drift space after the solenoid. This drift space is only $500 \mathrm{~mm}$ long, so magnets with an effective length of $0.1 \mathrm{~m}$ and a gradient of $1.0 \mathrm{~T} / \mathrm{m}$, similar to those in the electron linac, would be used and could be installed without major disruption.

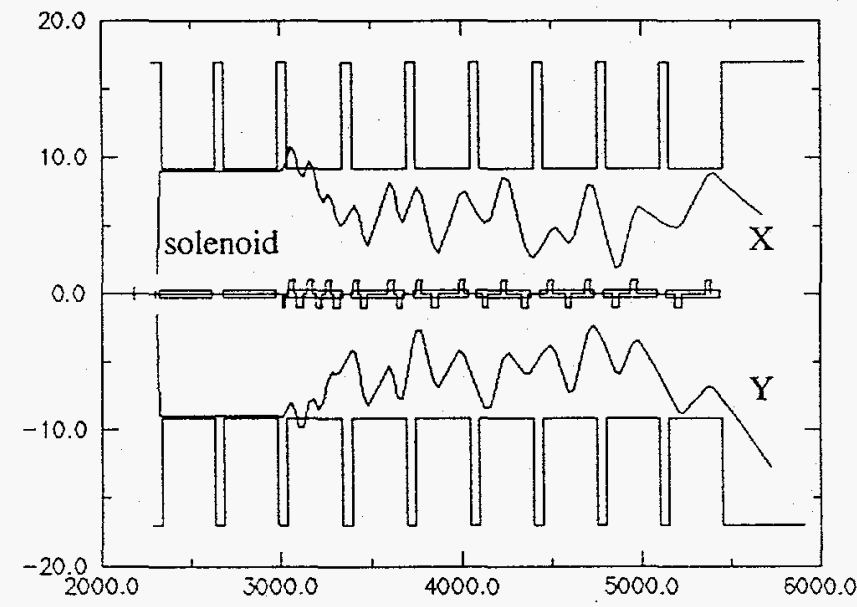

Figure 3: Calculated positron envelope with the existing focusing system.

The calculated positron linac beam envelope with a possible modified focusing scheme is shown in Figure 4. The additional doublet reduces beam losses caused by the downstream quadrupole magnets and significantly reduces beam profile expansion in the first drift space after the solenoid. The 38-mm-diameter vacuum pipe in the drift space allows the quadrupoles to function without causing losses, and it reduces the transverse beam size at the entrance to the next accelerating structure, so that downstream quadrupoles do not cause further beam losses.

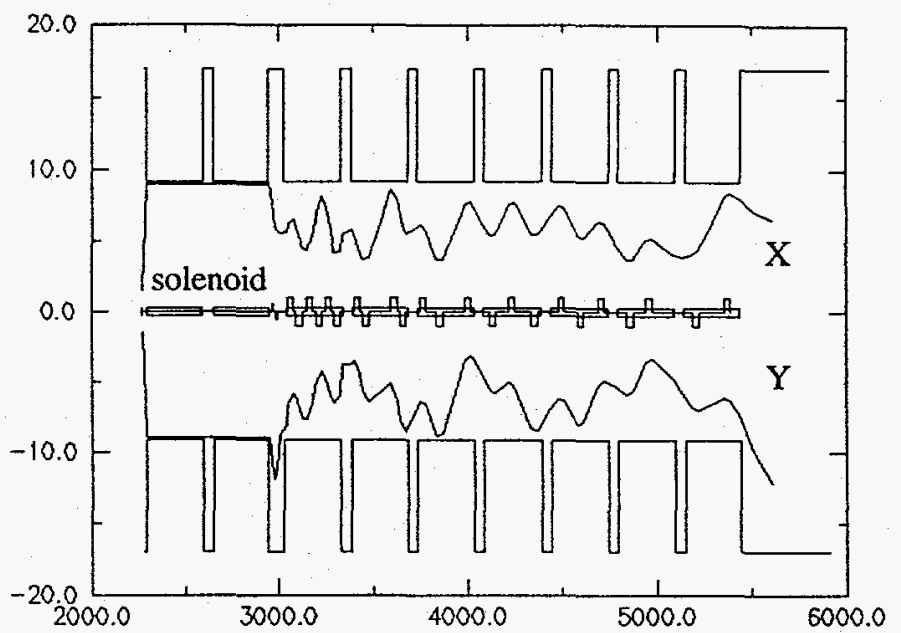

Figure 4: Calculated positron envelope with a possible modified focusing system.

\section{CONCLUSION}

The APS linac positron focusing system is adequate to provide the design positron current; however, a few simple modifications might be done that could significantly improve the performance and make the positron linac much easier to optimize.

\section{REFERENCES}

[1] M. White, et al., "Performance of the Advanced Photon Source Linear Accelerator," these proceedings.

[2] Coors Ceramic, Inc.

[3] G. Stange, IEEE Trans. Nucl. Sci. NS-26 4146 (1979).

[4] L. Rinolfi, "The Positron Production at LIL," ESRF Workshop, Grenoble (1992).

[5] The Stanford Two Mile Accelerator, Chap. 16, Benjamin, New York (1968).

[6] S. Humphries, Jr., Charged Particle Beams, (John Wiley and Sons, Inc., New York, 1990), p. 402.

[7] A. Enomoto et al., "Focusing System of the KEK Positron Generator," Nucl. Inst. and Methods, A281 19 (1989).

[8] TRANSPORT Code, SLAC-91 Rev. 2.

\section{DISCLAIMER}

This report was prepared as an account of work sponsored by an agency of the United States Government. Neither the United States Government nor any agency thereof, nor any of their employees, makes any warranty, express or implied, or assumes any legal liability or responsibility for the accuracy, completeness, or usefulness of any information, apparatus, product, or process disclosed, or represents that its use would not infringe privately owned rights. Reference herein to any specific commercial product, process, or service by trade name, trademark, manufacturer, or otherwise does not necessarily constitute or imply its endorsement, recommendation, or favoring by the United States Government or any agency thereof. The views and opinions of authors expressed herein do not necessarily state or reflect those of the United States Government or any agency thereof. 